\title{
Contribución del ejercicio integrador simulado para la formación inicial de la competencia profesional registrar hechos económicos.
}

\author{
Contribution of the simulated integrating exercise for the initial training \\ of the professional competence of the economic facts recorder.
}

Elizabet Prado Chaviano. ${ }^{1}$, Ilvia Montalvo Palacios. ${ }^{2}$, Cecilia Celestrín Penabades. ${ }^{3}$ Liset Arencibia Díaz. ${ }^{4}$ \& Luis Efraín Velastegui López. ${ }^{5}$

DOI: https://doi.org/10.33262/visionariodigital.v5i2.1710

\begin{abstract}
The integrate exercises in the profiles of the graduated the accounting and finances career allow the nitration of content another components of the professional mode of action witch are externalize in the academic field through the process of teaching and learning of the subjects and contribute to the comprehensive training of learner. In this case, e proposal was made for simulated integrated exercise in the first year of the degree with the creation of hypothetical company and it is by ability, applying was learned in the subject of the year with and emphasis or recording the facts economics as a professional competence in their incial training from general accounting subject I and II of the accounting discipline.
\end{abstract}

Keywords. Professional competence, record economic facts

\section{Resumen}

Los ejercicios integradores en el perfil del licenciado de la carrera Contabilidad y Finanzas permiten la integración de contenidos y otros componentes del modo de

\footnotetext{
1 Profesora de la carrera Contabilidad y Finanzas. Universidad de Matanzas. Cuba. elizabetpradochaviano7979@ gmail.com, Orcid. http//://orcid.org/0000-0002-8101-0440

2 Profesora de la carrera Contabilidad y Finanzas. Universidad de Matanzas. Cuba. ilvia.montalvo@umcc.cu, ORCID: http://orcid.org/ 0000-0002-1938-7001

${ }^{3}$ Profesora de la carrera Contabilidad y Finanzas. Universidad de Matanzas. cecilia.celestrin@ umcc.cu, Orcid. http//://orcid.org/0000-0002-8719-069x

${ }^{4}$ Profesora de la carrera Contabilidad y Finanzas. Universidad de Matanzas. liset.arencibia@ umcc.cu, Orcid. http//://orcid.org/0000-003-1731-6899

${ }^{5}$ Ciencia Digital Editorial, Ecuador, luisefrainvelastegui@ cienciadigital.org
} 
actuación profesional, que se externalizan en el ámbito académico mediante el proceso de enseñanza aprendizaje de las asignaturas, y contribuyen en la formación integral de los educandos. En ese caso, se realiza una propuesta de un ejercicio integrador simulado en el primer año de la carrera, con la creación de una empresa hipotética y su viabilidad, aplicando lo aprendido en las asignaturas de ese año, con énfasis en el registro de los hechos económicos como una competencia profesional en su formación inicial desde las asignaturas Contabilidad General I y II de la disciplina Contabilidad.

Palabras claves. Competencia profesional, registrar hechos económicos

\section{Introducción}

Uno de los retos más significativos que hoy día se plantea la Educación Superior Contemporánea, es la formación de profesionales competentes que sean verdaderos transformadores de su entorno y comprometidos con el desarrollo social, de acuerdo a los constantes cambios que se originan en los escenarios ocupacionales y educativos (Prado, Baujín y Alonso, 2017 a ).

La formación del profesional en las Ciencias Contables y Financieras, ha sido una preocupación permanente de la Educación Superior Cubana en las últimas décadas, específicamente en la Contabilidad y Finanzas, donde se realiza de modo consciente y sobre bases científicas, para garantizar la preparación integral de los estudiantes universitarios, con el fin de lograr profesionales revolucionarios, cultos, competentes, independientes y creadores, para que puedan desempeñarse exitosamente en los diversos sectores de la economía y de la sociedad en general y en concordancia con lo declarado por la Junta de Acreditación Nacional (JAN), donde se plantea la necesidad de lograr en las universidades cubanas: "el egreso de profesionales con elevado nivel de calidad, independientemente de la modalidad de estudio de las carreras universitarias, con un desempeño ético, competente y transformador en la solución de problemas territoriales" (MES, 2018a, p. 5).

En este sentido, el estudio que se presenta, tiene como fin incorporar un ejercicio integrador simulado como vía de formación integral de los educandos, en el proceso de enseñanza aprendizaje del primer año de la carrera Contabilidad armonizado con las exigencias del Plan de Estudio E.

\section{Desarrollo}

EL ejercicio integrador ha sido considerado una de las vías más efectivas para lograr un aprendizaje desarrollador en la carrera Contabilidad y Finanzas, muestra de eso, es su aplicación como parte de la estrategia en la disciplina Contabilidad en los diferentes planes de estudio, con énfasis en la demostración del registro de los hechos económicos de forma manual y en software (Prado y López, 2020).

El registro de los hechos económicos mencionado anteriormente, se aborda como una competencia profesional específica del contador en entornos como Perú, con un perfil de 
competencias laborales que presenta como elementos que caracterizan dicha competencia profesional, el código de la norma y elemento de la competencia, los conocimientos fundamentales, los criterios de desempeño vinculados a la preparación de los asientos de diarios, según plan contable y los estados financieros, según políticas establecidas (SINEACE, 2014).

Este sistema se limita a la confección de perfiles laborales para los especialistas de Gestión Económica Financiera y cuentan con las exigencias establecidas para la contratación y la evaluación de desempeño en el puesto de trabajo, y aunque consideran los conocimientos y habilidades en contexto laboral, no establecen las pautas para la formación de esta competencia profesional específica en el proceso de enseñanza aprendizaje en las universidades.

Para Vázquez y Saitua (2007) de la Universidad Euskal Herriko Unibertsitatea del país Vasco, la competencia registrar hechos económicos es una de las competencias profesionales a desarrollar desde la asignatura Contabilidad, y lo basa en los requerimientos dictaminados en los programas de Asesoramiento a la Introducción del Crédito Europeo y de Seguimiento para la Introducción de los Créditos Europeos y mencionan dentro de las acciones a desarrollar las clases y exámenes teórico-prácticos, pruebas teóricas y trabajos prácticos grupales. En ese sentido, a pesar de mencionar actividades en función de esa competencia profesional, no se trazan las estrategias metodológicas que permiten esa formación. No se describen los contenidos, los medios y métodos, ni la bibliografía empleada en estas actividades, así como las vías o procedimientos para la constatación del desempeño desde el proceso de enseñanza aprendizaje y en el contexto laboral.

Autores como Ríos, Galache, Dieguez y Sánchez (2012), tienen en cuenta en la guía de la asignatura Contabilidad II, diferentes tipos de entidades, la normativa contable, las cuentas y su registro en los libros obligatorios, apoyados en sistemas contables automatizados; la utilización de casos de estudio, las tutorías y consideran los componentes del proceso de enseñanza aprendizaje, sin embargo, no tratan las regularidades de las cuentas de los hechos económicos para cualquier tipo de entidad, los documentos mercantiles, los hechos económicos y su tipificación, por otra parte los métodos de enseñanza expresados son limitados al igual que la evaluación que prioriza el examen escrito.

También el Sistema Nacional de Aprendizaje de Colombia SENA (2017) trata el registro de hechos económicos, como una competencia mínima a desarrollar, pero no hace alusión a la tipificación de los hechos económicos y las regularidades de las cuentas del registro de los hechos económicos y menciona los deberes y derechos del contador y no conciben esta competencia profesional como la integración de conocimientos, habilidades, valores, motivación y desempeño donde intervienen los profesores, tutores, estudiantes y otros contextos de actuación. 
El análisis realizado de los criterios expuestos, les permite a los autores considerar la competencia profesional específica registrar hechos económicos, como un proceso continuo, complejo y sistémico que integra conocimientos, habilidades, valores éticos del contador, motivaciones y desempeños relacionados con el análisis y procesamiento de los hechos económicos para su posterior registro en los libros obligatorios de la contabilidad acorde con la normativa contable evidenciados en la actividad académica y el entorno laboral con la ayuda del profesor, el tutor y otros contextos de actuación.

La formación inicial de la competencia profesional específica registrar hechos económicos ocurre en los dos primeros años mediante el proceso de enseñanza de la disciplina Contabilidad, donde se registran los hechos económicos en diversas entidades en las asignaturas Contabilidad General I y II y se desarrollan en el estudiante los modos de actuación profesional a través de la disciplina integradora en ese caso con el ejercicio integrador simulado en el primer año de la carrera. En el ejercicio integrador simulado se tienen en cuenta diferentes aspectos, tales como, la normativa contable, la documentación mercantil, los libros de contabilidad, las cuentas y subcuentas y contabilizar los hechos económicos en el libro diario y los procedimientos contables (Rajadell, Trullás y Simo, 2014).

\section{Métodos}

Para la elaboración del ejercicio integrador simulado se realizó como parte de un estudio precedente, un diagnóstico sustentado en la utilización de un conjunto de métodos que a continuación se detallan.

Revisión de documentos: se revisaron 7 ejercicios integradores aplicados para el primer año de la carrera, en los cursos 2011-2012, 2012-2013, 2013-2014, 2014-2015, 20152016, 2017-2018 y 2019 -2020, donde se constató la presencia de los contenidos de las asignaturas del año, para lo cual se realizó la tabla 1 que está compuesta por el número de ejercicios, la asignatura y los contenidos. El número consecutivo del 1 al 7 corresponde al orden de los cursos mencionados anteriormente.

Tabla 1. Relación entre los ejercicios y contenidos por asignaturas del año

\begin{tabular}{ccc}
\hline Ejercicios & $\begin{array}{c}\text { Asignaturas } \\
\text { incluidas }\end{array}$ & Contenidos \\
\hline
\end{tabular}

1 y 2 Contabilidad General I 1. Los Registros Básicos y Auxiliares de la Contabilidad.

2. Los Estados Financieros Básicos: Objetivos y presentación de las partidas que lo integran.

3. Técnicas de valuación, registro y presentación

Contabilidad General de las partidas fundamentales que integran el II Activo Circulante.

4. Documentos Mercantiles,

5. Inventario. Tipos. Valoración, recargos y descuentos comerciales.

6. Las mercancías en Comisión y Consignación. 
Nota: en el caso del ejercicio 2 tiene presente además la asignatura de Matemática Financiera que se presentan en la columna que se encuentra a la derecha.

3 al 7 Contabilidad General I 1. Los Registros Básicos y Auxiliares de la Contabilidad.

2. Los Estados Financieros Básicos: Objetivos y presentación de las partidas que lo integran.

Contabilidad General 3. Técnicas de valuación, registro y presentación de II

las partidas fundamentales que integran el Activo Circulante.

4. Documentos Mercantiles

5. Inventario. Tipos. Valoración, recargos y descuentos comerciales.

6. Las mercancías en Comisión y Consignación.

7. Registro de una operación en los libros de diario

Inglés en este idioma.

8. Traducción del resumen del trabajo.

Derecho 9. Proceso de Litigio desde la participación de un jurídico.

Fuente: elaboración propia

Una vez analizado los 7 ejercicios se considera la presencia de 5 asignaturas fundamentalmente, Contabilidad General I y II, Derecho, Inglés y Matemática Financiera que representa el $21 \%$ del total de las asignaturas del año y el incumplimiento de los objetivos previstos desde las asignaturas en concordancia con el año académico, exceptuando solo el caso del objetivo 1 relacionado con la aplicación de las técnicas de registro y presentación de la información económico - financiera, este con la presencia de los registros de hechos económicos y la elaboración de los estados financieros, por lo cual se considera insuficiente su tratamiento desde la competencia profesional registrar hechos económicos, porque existe una omisión de aspectos relacionados con documentos mercantiles, procedimientos contables, las cuentas, tipos de organizaciones y el registro de los hechos económicos en software así como las relaciones interdisciplinarias desde todas las asignaturas del año, donde se considera apropiado insertar la vinculación de la política de la revolución cubana para enfrentar las situaciones excepcionales y la importancia de las tradiciones patrióticas en la defensa de la nación cubana. La propuesta puede estar incluida por las asignaturas de Defensa Nacional e Historia de Cuba, así como las relacionadas con los contenidos de Economía Política y el registro de los hechos económicos relacionados con procesos de litigio con la presencia de la asignatura de Derecho.

Encuestas: aplicadas a los 7 profesores del colectivo de año, para de determinar los contenidos y objetivos a cumplir en correspondencia con las asignaturas del año, lo cual se presenta en la tabla 2 que está compuesta por las asignaturas, contenidos y escalas de 
evaluación con las categorías Incluido (I) y Parcialmente incluido (PI). A continuación se presenta una muestra de la asignatura Derecho.

Tabla 2. Muestra del análisis realizado para los objetivos principales

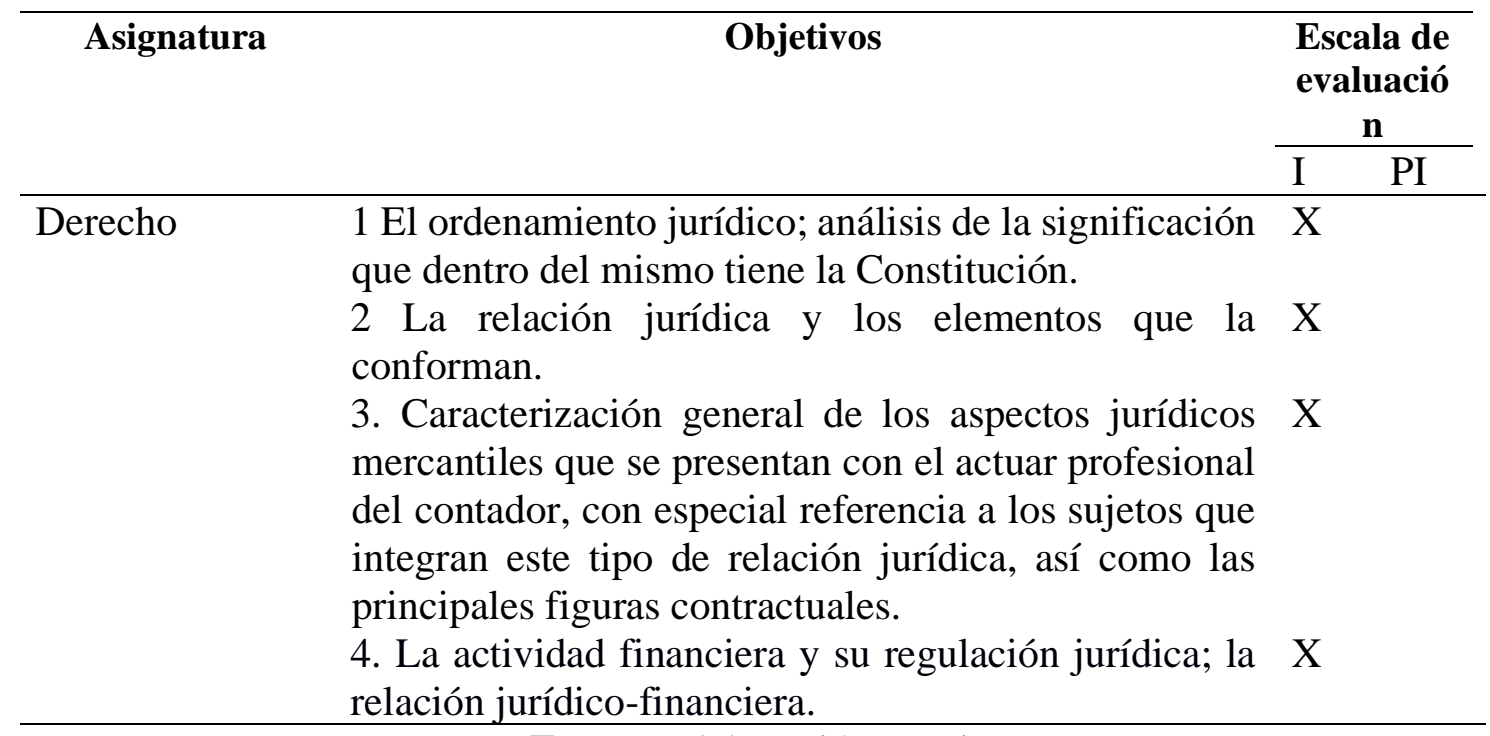

Fuente: elaboración propia

Entrevista no estructurada: se le realizó a los mismos profesores que se mencionan anteriormente con el objetivo de determinar la propuesta de integración de cada asignatura dentro del ejercicio integrador simulado. Cómo resultado se obtuvo para cada una de las temáticas los ejemplos de cómo integrar el contenido y notas aclaratorias dónde se le explica a los estudiantes cuales pueden ser las bases para realizar la integración del contenido. En caso de los registros contables se presentan ejemplos de operaciones para asegurar que no le falte información teniendo en cuenta la actividad empresarial real.

Teniendo en cuenta lo anteriormente planteado, los autores consideran que se debe agregar contenidos de las demás asignaturas del año como Derecho, Defensa Nacional, Inglés e Historia de Cuba, así como las relacionadas con los contenidos de Economía Política por lo que a continuación se muestra la propuesta de la guía del ejercicio integrador simulado y se define como objetivo del mismo: resolver problemas de la profesión apoyado en los conocimientos de las asignaturas que integran el año, con énfasis en el registro de los hechos económicos como competencia profesional del contador.

La orientación comprende de una introducción donde se presentan las bases fundamentales del Plan de Estudio E, la importancia y el objetivo del ejercicio integrador simulado.

El ejercicio integrador simulado consta de cuatro partes:

En la parte I, se definen como aspectos generales para la creación del ejercicio integrador simulado que el estudiante debe ser capaz de crear su propia empresa, esta puede ser una entidad estatal o un negocio particular. La orientación que se muestra más adelante se 
relaciona directamente con una entidad estatal, no obstante, se explica algunas consideraciones para realizar el caso del negocio particular.

El ejercicio tiene que ser integrador por lo que debe abarcar contenidos del $80 \%$ de las asignaturas recibidas en el curso.

1. Se puede desarrollar por equipos de dos estudiantes (como máximo) o individual.

2. El ejercicio integrador debe entregarse por escrito ya sea usando las tecnologías de la información y las comunicaciones o manuscrito, manteniendo durante todo el trabajo la limpieza y detalles de presentación de la información contable. (Ver sugerencias sobre la estructura del informe en la parte II)

3. Se defiende ante un tribunal el cual debe estar constituido por profesores de dichas asignaturas. En la defensa debe demostrar dominio del trabajo y de los contenidos que abarca.

4. Se deben realizar controles parciales por parte de los profesores de la asignatura de manera que permita un seguimiento del ejercicio, así como aclaraciones de dudas. (Ver sugerencias sobre la estructura del informe en la parte III)

5. El ejercicio integrador es un ejercicio hipotético que tiene fines docentes por lo que contempla un grupo de operaciones que todas ellas se realizan en el período de un mes y termina con el cierre contable, partiendo del criterio que son hechos económicos que simulan las operaciones de una empresa hipotética, con el fin de facilitar el trabajo de los estudiantes. El profesor tiene la potestad de variar el período así como los importes del Estado de Situación inicial manteniendo como mínimo las operaciones que se indican.

6. El trabajo no debe presentarse como una relación de preguntas y respuestas. Propuesta de integración por cada asignatura

La propuesta de integración se realiza mediante un taller de corte y costura imaginario donde al estudiante se le ejemplifican con las asignaturas del año y la vinculación con el registro de os hechos económicos.

\section{Asignatura Derecho}

Nota: el estudiante debe de ser capaz de crear el objeto social de su empresa estatal o su propio negocio.

Ejemplo de objeto social

No necesariamente el estudiante tiene que poner la misión y visión.

Objeto Social

- Prestar Servicios de corte y costura.

- Producir y comercializar productos elaborados de vestuario.

- Brindar servicio de capacitación y adiestramiento al personal del taller. 
Misión: el taller de corte y costura brinda servicios con calidad a un costo asequible para satisfacer las necesidades de los clientes, apoyados en la profesionalidad de su capital humano.

Visión: somos líderes en el mercado de corte y costura por su calidad en los productos, su precio asequible y la profesionalidad de su capital humano.

Nota: que el estudiante pueda definir quiénes son los miembros que conforman el órgano de justicia laboral de base .Presidente, secretario, vocal y suplentes.

Ejemplo: la administración del taller decide aplicarle una sanción a un trabajador afectándole su estimulación por valor de \$ --------. Dicha medida estaba mal aplicada, el trabajador acude al órgano justicia laboral de base para ser su reclamación, el órgano falla a favor del mismo.

\section{Asignatura Seguridad y Defensa Nacional}

El diferendo EEUU-Cuba no es más que las relaciones diplomáticas entre ambos países. Una de las manifestaciones que se hace visible en la actualidad el bloqueo económico impuesto a Cuba.

Nota: el estudiante tiene que ser capaz de analizar dentro de su empresa cuales pueden ser las afectaciones debido recrudecimiento de bloqueo y la incidencia en el registro de los hechos económicos.

Ejemplo: producto al recrudecimiento del bloqueo económico de EEUU hacia Cuba. El proveedor del taller de corte y costura, compra materias primas y materiales a través de un tercer país cuyo valor inicial es de $\$$----- pagando intereses del ---\% al otro país. Tiene que pagar aranceles por valor de $\$$----- por estadía en el puerto durante 2 días.

Nota: el estudiante tiene qué ser capaz de definir los productos que la empresa tiene bajo su custodia para situaciones excepcionales.

Ejemplo: el económico de la empresa decide hacer un inventario sorpresivo al almacén del taller de corte costura con el objetivo de controlar las reservas movilizativas la actividad fue realizada a través de un conteo físico que abarcó siendo su universo el 100\%.

\section{Asignatura Historia de Cuba}

Nota: el estudiante debe explicar las distintas conclusiones de los congresos del Partido Comunista de Cuba para saber la trayectoria económica política del país.

Ejemplo: el conocimiento los lineamientos del Vll del PCC y la vinculación con el registro de los hechos económicos.

\section{Asignatura Inglés 1}

Nota: que el estudiante sea capaz de realizar una traducción de alguna parte del ejercicio y además la exposición de algún registro contable. 
Ejemplo: traducir el resumen en ese idioma.

Registrar un hecho económico con todos los detalles en este idioma y además su exposición.

\section{Asignatura Economía Política}

Nota: el estudiante tiene qué ser capaz de definir qué es planificación y mercado, y la relación que existe entre ambos. Se puede destacar que el cliente más significativo es el Ministerio de Salud.

Ejemplo: el taller de corte y costura dentro de su plan tiene conveniado entre otras producciones batas con destino a salud.

Nota: el estudiante tiene qué ser capaz de definir las diferencias existentes entre producir y distribuir y que entre ambas relaciones existe un convenio.

Ejemplo: el taller de corte y costura produjo ---- cantidad de batas sanitarias y otras producciones al Ministerio de Salud.

\section{Asignatura Matemática Financiera}

Nota: el estudiante tiene qué ser capaz de definir los métodos para el cálculo del monto y del interés simple. Diferentes tipos de intereses y la vinculación con el registro de los hechos económicos.

Ejemplo: el taller de corte y costura vende a plazos un producto y utiliza el método para el cálculo del interés.

Nota: el estudiante tiene que ser capaz de definir la fórmula del monto y del interés compuesto ara el posterior registro de los hechos económicos.

Ejemplo: la empresa a la que pertenece el taller de corte y costura, acude al banco por un préstamo para la compra de materia prima y materiales. Teniendo que pagar intereses del --------\%

\section{Asignatura Contabilidad General I}

Nota: que el estudiante sea capaz de definir que son los registros básicos y auxiliares de la Contabilidad, describir como se evidencian las Normas Cubanas de Contabilidad e Internacionales de la Información Financiera, clasificar los hechos económicos que registró, registrar los mismos ya sea de forma manual o en software con las cuentas correspondientes teniendo en cuenta las regularidades de las cuentas en el registro de los hechos económicos de diversas entidades.

Ejemplo: el taller de corte y costura

Ejemplo: el taller de corte y costura presenta por su nivel de producción 1000 unidades, realiza como parte de las compras correspondientes a este período, el día 5 compra de 
Materiales y Materias Primas a los proveedores Cimex por \$_y Prestal \$_ amparados en el primer caso por la Factura No. 25 y en el segundo por la Letra de cambio 7895 por lo que se debe registrar en los diarios auxiliares de compras.

Norma contable que se cumple:

Tipos de hechos económicos: compra, venta, cobro, pago, devolución

Nota: que el estudiante sea capaz de presentar la información contable y financiera en los estados financieros Básicos

\section{Asignatura Contabilidad General II}

Nota: que el estudiante sea capaz de registrar y presentar las partidas fundamentales que integran el Activo Circulante apoyado en procedimientos contables

Ejemplo: el taller de corte y costura

Procedimientos contables: se tienen en cuenta los objetivos del procedimiento ejemplo: establecer los pasos para el control y contabilización de los Útiles y Herramientas en almacén y los de uso.

El alcance del procedimiento ejemplo es asociado al control y contabilización de los Útiles y Herramientas.

Los documentos de referencia tienen como objetivo describir las resoluciones que amparan el procedimiento ejemplo resoluciones asociadas al control y contabilización de los Útiles y Herramientas.

- Resolución No. 060/11, Contraloría General República, de fecha 01/03/2011

- Resolución No. 235/05, Ministerio Finanzas y Precios, de fecha 30/11/2005

- Resolución No. 268/18, Ministerio Finanzas y Precios, de fecha 22/4/2018

- Resolución No. 1038/17, Ministerio Finanzas y Precios, de fecha 25/12/2017

El control Interno. Tiene como propósito determinar los pasos a seguir ejemplo con los pasos para la recepción de Útiles y Herramientas, ya sea cuando se produzcan altas, bajas o devoluciones como los que son puestos en explotación para llevar un control adecuado de los mismos.

Ejemplo: la recepción de los Útiles y Herramientas en el almacén debe realizarse por el Modelo SC-2-04 Informe de Recepción, el control dentro del almacén se realiza por el Modelo SC-2-14 Tarjeta de Estiba, la salida del almacén por el modelo SC-2-08 Vale de Entrega o Devolución, según se establece en el Procedimiento 07-03-01 Procedimiento General de Inventarios.

El registro y modelos asociados de uso obligatorio. Hace referencia a los modelos asociados al procedimiento control y contabilización de los Útiles y Herramientas. 
Ejemplo:

- Registro No. 07-03-01 General de Inventario

- Registro No. 07-03-03 Ajuste de Faltantes y Sobrantes de Inventario

- SC-2-04 - Informe de Recepción

- SC-2-08 - Vale de Entrega o Devolución

- SC-2-12- Factura

- SC-2-13 - Submayor de Inventarios

- SC-2-14 - Tarjeta de Estiba

- SC-2-18 - Factura Comercial

- MC-2-01 - Control de Útiles y Herramientas

Las responsabilidades consiste en delimitar las responsabilidades por área con respecto al procedimiento específico control y contabilización de los Útiles y Herramientas.

Ejemplo:

- Aprobación: Director Contable-Financiero

- Actualización: Jefe del Grupo de Contabilidad

- Implementación: Directores

- Supervisión: Comité de Control y Auditores Internos de la Empresa

El archivo establece las condiciones del archivo de los registros así como el soporte que será utilizado para su custodia e impedir su deterioro y el período en el cual permanecerán archivados los mismos.

Ejemplo: los documentos serán archivados por un término de cinco (5) años a partir de la fecha de cierre del ejercicio contable. (Resuelvo Octavo, Resolución No. 54/05, MFP). Las condiciones del archivo de los documentos de las acciones preventivas tomadas ya sea en soporte magnético o escrito serán las adecuadas para su custodia e impedir su deterioro. Igualmente estarán clasificados y ordenados de forma que sea fácil su utilización, protección y recuperación. Las entidades en su procedimiento deben definir en qué legajo se archiva, así como su localización física.

La fecha de vigencia, se propone la fecha según la cual tendrá vigencia el procedimiento antes descrito.

Ejemplo: este procedimiento tendrá vigencia a partir del _ de noviembre del

Fase 2. Registro de los hechos económicos asociado al control de Útiles y Herramientas en uso.

Etapa 1. Definir el tipo de hecho económico relacionado con el Inventario.

1. Por la recepción de Útiles y Herramientas, cuando la compra se realiza al crédito. Adquisición.

2. Por la recepción de Útiles y Herramientas, cuando el pago se ha realizado adelantado. Adquisición. 
3. Por la salida del almacén y recepción por el área, pañol o el trabajador para su puesta en explotación o en uso. Transferencia entre áreas.

4. Por el gasto del $50 \%$ de su valor de adquisición. Gasto.

Determinación de las cuentas que intervienen. Para lo cual se proponen tres tareas:

Tarea 1. Identificación de las cuentas que se afectan.

- Por la recepción de Útiles y Herramientas, cuando la compra se realiza un débito a la cuenta de Útiles y Herramientas y crédito a Cuentas por Pagar a Corto Plazo.

- Por el gasto del $50 \%$ de su valor de adquisición: intervienen al débito el Gasto que Corresponda y al crédito Desgaste de Útiles y Herramientas.

Tarea 2. Clasificación de las cuentas que se afectan.

\begin{tabular}{lll}
\hline \multicolumn{1}{c}{ Cuentas Reales } & \multicolumn{2}{c}{ Cuentas Nominales } \\
\hline Útiles y Herramientas & Cuenta Gasto que \\
Cuentas por Pagar a Corto Plazo & corresponda & \\
Faltante de Bienes en Investigación & & \\
Sobrante de Bienes en Investigación & \\
Pagos anticipados a suministradores & \\
\hline
\end{tabular}

Tarea 3. Determinación de las Subcuentas y Análisis.

- Por la recepción de Útiles y Herramientas, se utilizan las Subcuentas: en Almacén (187. 0010), subcuenta que corresponda y Análisis por Suministrador.

- Por el gasto del $50 \%$ de su valor de adquisición: intervienen las subcuentas: subcuenta que corresponda.

Contabilización. Para la cual se proponen cuatro tareas:

Tarea 1. Utilización del Libro de Diario.

Formato del libro de Diario General

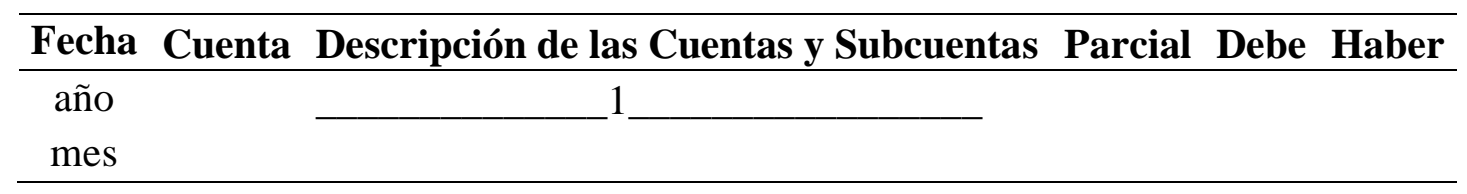

Tarea 2. Ubicación de los datos preliminares con el fin de representar la fecha, nombre de la empresa, el número de asientos de diario, el folio y año.

Empresa

1. Por la recepción de Útiles y Herramientas, cuando la compra se realiza al crédito.

2 . 


\begin{tabular}{ccc}
\hline Fecha & Folio & No asiento \\
\hline $\begin{array}{c}\text { noviembre } \\
1\end{array}$ & 1 & -1 \\
\hline
\end{tabular}

Tarea 3. Ubicación de las cuentas en él Debe y el Haber en el comprobante y Asientos de Diario.

1. Por la recepción de un útil o herramienta, cuando la compra se realiza al crédito.

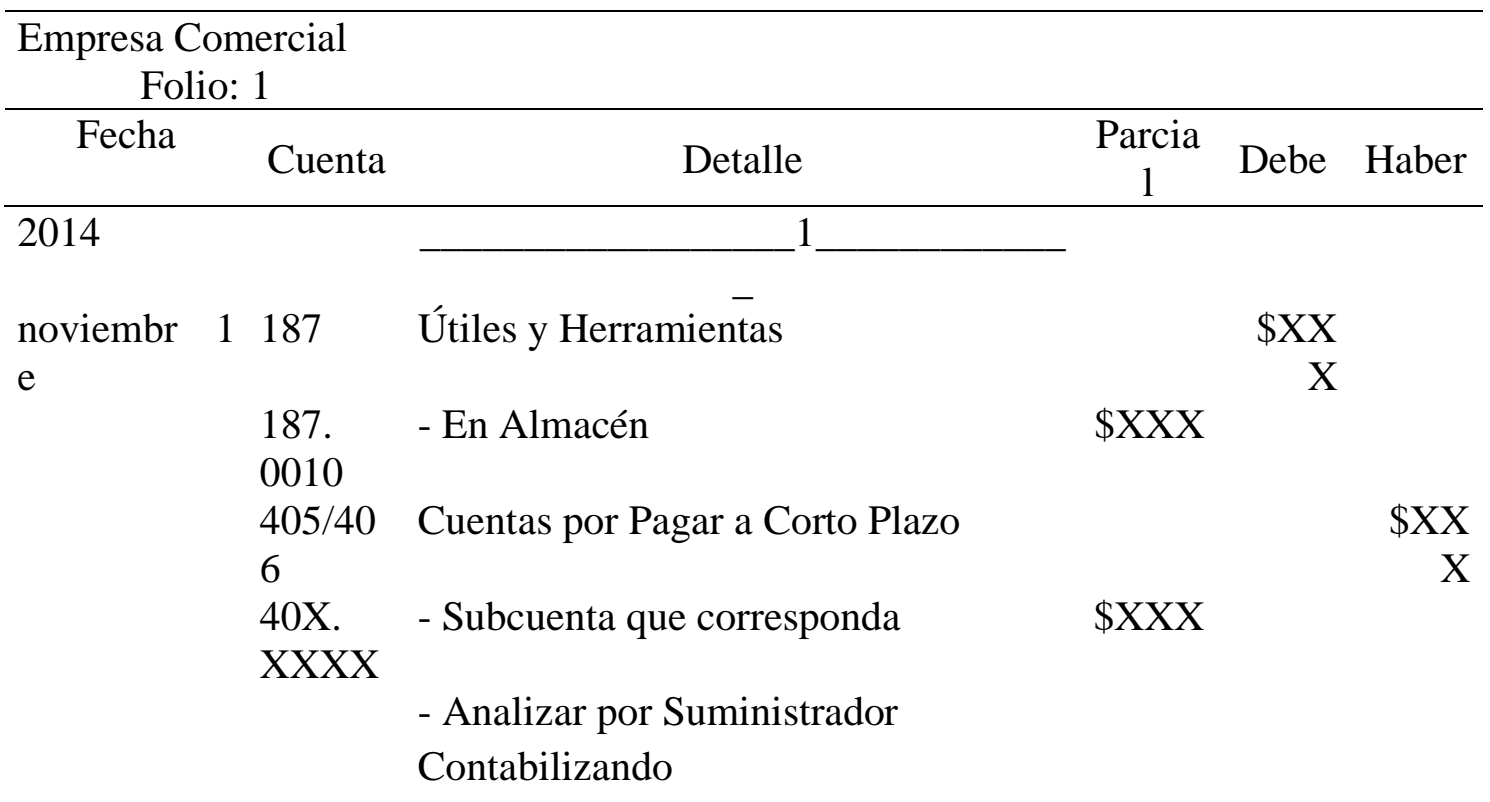

A continuación se presentan otros ejemplos de hechos económicos estudiados.

Fecha No. Hechos económicos acontecidos en el mes

1. Se deposita en el Banco el importe de la cuenta Efectivo en Caja Fondo para Depositar con Depósito NO.

2. Compra de mercancías al crédito y al contado emitiendo Cheque No.___ (especificando tipo de mercancía, cantidad de unidades y precio unitario y nombre del proveedor y condiciones de crédito $(2 / 10 \mathrm{~N} / 30)$

3. Se pagan los sueldos acumulados emitiéndose el cheque No.

4. Liquidación de la cuenta por pagar de uno de los acreedores con Cheque No.

5. Se paga Fletes en compras a Nombre del transportador con Cheque No.

6. Se amplía el Fondo para Pagos Menores según Cheque No.

7. Se recibe nota de crédito del banco notificando el cobro del documento enviado para esta gestión del cliente , con deducción de comisión bancaria.

8. Se toma el descuento por pronto pago de la primera operación de compra realizando el pago en los días acordados. 
9. Venta de mercancías recibiendo Letra de Cambio (especificando tipo de mercancía, cantidad de unidades y precio unitario y nombre del cliente y período de crédito)

10 Cancelar como incobrable la Cuenta por Cobrar de un cliente con cuenta ya vencida (tenga en cuenta la información inicial sobre clientes)

11 Se pagan fletes en ventas a Nombre del transportador mediante el Fondo para Pagos Menores (se emite el vale o comprobante).

12 Se cobra el primer plazo del cliente (Submayor de Cuentas por Cobrar a Plazos ) y deposita con Depósito No.

13 Se vence la Cuenta por Cobrar del cliente ___ y se renueva con una Letra de Cambio que genera intereses especificar el período de crédito así como si se incluye el interés en el valor de la letra o no).

14 Compra de mercancías al crédito mediante Efectos por Pagar (especificando tipo de mercancía, cantidad de unidades y precio unitario y nombre del proveedor y período de crédito).

15 Se descuenta el documento del cliente en el Banco a una tasa de interés del

16 Se vende mercancía al contado al cliente recibiéndose el cheque No. (especificando tipo de mercancía, cantidad de unidades y precio unitario y nombre del cliente) el cual se deposita Depósito No.

17 Se pagan dietas de viaje del Fondo de Pagos Menores (se emite el vale o comprobante).

18 Se recupera la Cuenta por Cobrar del cliente cancelada con anterioridad.

19 Se compra mercancía al contado al proveedor emitiéndose el cheque No. (especificando tipo de mercancía, cantidad de unidades y precio unitario y nombre del proveedor).

En la parte II, sobre la elaboración del informe del ejercicio integrador debe contener los aspectos siguientes:

Portada: la misma recoge los elementos fundamentales que ubica el estudiante en la Universidad, facultad y carrera. Cómo título tendrá en cuenta ejercicio integrador simulado en la empresa $\mathrm{x}$. Los autores, así como la ciudad y año en que se realiza dicho trabajo.

Resumen: el mismo debe contener una síntesis de la importancia del trabajo el objetivo que persigue el mismo así como lo que se espera realizar en cada caso.

Nota: debe estar el resumen en inglés.

Introducción: se presentará la creación de la empresa o negocio acompañado de su objeto social con sus estructuras y funciones (al menos del área contable). Período de operaciones escogido, método de conciliación bancaria que se utilizará así como el método para el cálculo de las provisiones y el sistema de inventario utilizado. 
Desarrollo:

- El ejercicio puede partir o no de una información inicial tales como un Estado de Situación o Balance de Comprobación, como base para la posterior incorporación de operaciones económicas que deberán ser elaboradas (creadas) por cada estudiante o equipo de estudiantes integrando los contenidos de las asignaturas del año.

- Se deben presentar el total de operaciones del mes así como la interrelación de conocimientos simulados a partir de la propuesta que se encuentra en la parte I.

- Seguidamente se sugiere presentar los registros de diario, pases al mayor, elaboración del balance de comprobación, registro de ajustes contables y correcciones de errores, balance de comprobación ajustada, así como los estados financieros y asientos de cierre.

Nota: es importante que todos los estudiantes tengan en cuenta la relación que se debe mostrar entre los contenidos de las diferentes asignaturas del año.

Conclusiones: deben estar encaminadas al cumplimiento de los objetivos del trabajo y la importancia que ha tenido para el estudiante este tipo de ejercicio. Así como los principales resultados del ejercicio integrador simulado.

Bibliografía: se incluye toda la utilizada para la realización del trabajo.

Anexos: pueden ubicarse estados de cuentas del banco, estado de conciliación bancaria, vales de caja chica y otros elementos que permitan la comprensión del presente trabajo.

En la parte III sobre el cronograma de orientación y control sistemático, se realiza en función de las actividades, por lo tanto, se ilustra en la tabla 3 que se presenta a continuación:

Tabla 3. Cronograma de trabajo

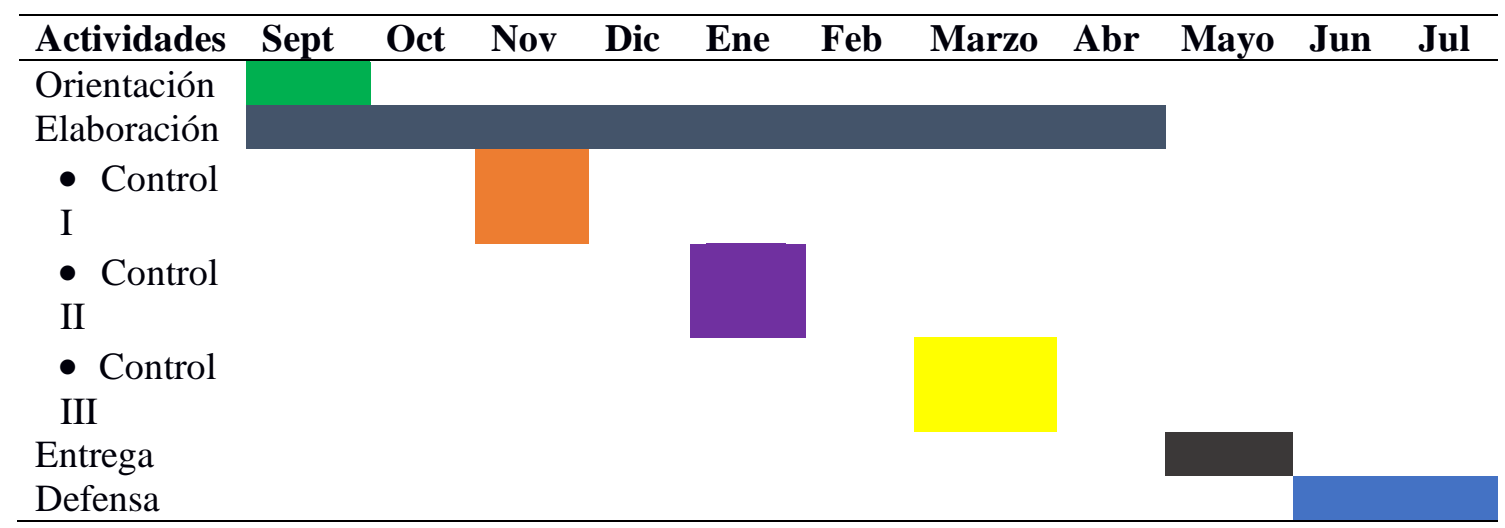

Fuente: elaboración propia

La fecha exacta de los controles será propuesta por los profesores del año. Aunque se recomienda para ello tener en cuenta los días que se planifican por la facultad de trabajo científico estudiantil. 
En la parte IV se realizan las orientaciones sobre la presentación frente a un tribunal y se recomienda que sea en PowerPoint o en pancartas.

Presentación

Introducción: (cuatro diapositivas máximo que tengan: Importancia del trabajo y la caracterización del negocio o empresa que crea)

Desarrollo: (deben presentarse el ciclo contable en el período que se realizó)

Conclusiones

Recomendaciones

Las diapositivas deben tener colores claros y letra oscura, letra Arial 20, no cargadas de letras.

Importante: para la evaluación de este ejercicio se tienen en cuenta:

Entrega en las etapas establecidas las tareas del ejercicio.

Apreciación del interés y disposición en la solución del mismo a partir de las limitaciones y logros en el aprendizaje.

Valores éticos de la profesión como laboriosidad, responsabilidad y trabajo en equipo.

\section{Resultados}

El $100 \%$ de los profesores estuvieron presentes en la defensa de este ejercicio, y consideraron que permitió la integración de conocimientos, habilidades, motivación, valores y desempeños mientras que todos los estudiantes realizan equipos de dos participantes, donde el $23.07 \%$ crean entidades de servicios, el $46.15 \%$ crean entidades comerciales y el resto el $30.76 \%$ crean entidades industriales, lo que demuestra la consolidación de los aspectos sobre los hechos económicos. En la evaluación sistemática se tuvieron en cuenta las limitaciones y progresos en el aprendizaje, el interés, el trabajo en equipo, las relaciones interpersonales que establecen, la manifestación de los valores éticos del contador y los conocimientos, habilidades y desempeño demostrado en el desarrollo del ejercicio, que al ser una simulación, constituye un antecedente para la práctica laboral.

El $64.61 \%$ de los estudiantes entregaron todas las tareas según el cronograma establecido y mostraron un buen desempeño en la formación inicial de esta competencia profesional específica, el resto presentó algunas limitaciones, esencialmente en los hechos económicos, relacionados con las situaciones excepcionales y la motivación por mejorar la calidad en la presentación del ejercicio. 


\section{Conclusiones}

- Se constata la aplicación de los elementos a tener en cuenta desde la teoría en torno a las relaciones interdisciplinarias, la integración de conocimientos, habilidades, motivaciones, valores y desempeños en el entorno académico desde las asignaturas del año, con énfasis en el registro de los hechos económicos, los procedimientos contables, documentos mercantiles, las cuentas y las organizaciones.

- El diseño del ejercicio integrador simulado propició un perfeccionamiento en el trabajo metodológico del año y un acercamiento a la práctica contable como antecedente para la futura práctica laboral.

- El control sistemático y presentación del trabajo contribuyeron en el desempeño demostrado teórico-práctico y argumentativo de los aspectos considerados desde la formación inicial de la competencia profesional registrar hechos económicos.

\section{Referencias bibliográficas}

MES. (2018a). Resolución Ministerial No. 150/2018. Reglamento del Sistema de Evaluación y Acreditación de la Educación Superior. La Habana (Cuba).

Prado, E., Baujín, P., y Alonso, C. (2017a). La multidisciplinareidad para la formaciòn en los estudiantes de la carrera Contabilidad y Finanzas en el registro de hechos econòmicos. Revista del instituto Pedagógico Latinoamericano y Caribeño, 6(12), sp. Recuperado de: http//revista.iplac.rimed.cu.

Prado, E., Díaz, A., y Montalvo, I. (2015). Procedimiento que contribuye al desarrollo de la interdisciplinaridad en la carrera Contabilidad y Finanzas apoyado en el registro de hechos económicos. R, Vizcon (Presidencia), Conferencia metodológica. Conferencia dirigida por la Universidad de Matanzas, Matanzas

Prado, E., Pérez, M., y Montalvo, I. (2020). La formación de la competencia registrar hechos económicos apoyado en la informática. Evento Provincial del Maestro. Evento dirigido por la Universidad de Matanzas.

Prado, E., y López, L. (2020). El registro de los hechos económicos en la actividad comercial, como punto de partida en la enseñanza de la Auditoría. Revista de Auditoría y Control, 40(1), p. 94-100. Recuperado de: http//contraloría.gob.cu.

Rajadell, M., Trullás, O., y Simo, P. (2014). Contabilidad para todos. Introducción al registro contable. España. Editorial Omnia Publisher.

Ríos, M., Galache, T., Dieguez, J., y Sánchez, R. (2012). Guía docente de la asignatura Contabilidad General II. Revista de Ciencias Económicas y Empresariales, 8(1), p. 1-4. Recuperado de: http//www.uma.es.

SENA. (2017). Sistema Nacional de aprendizaje. Bogotá. Cundinamarca. Colombia. Recuperado de: http://sena.edu.cu. 
SINEACE. (2014). Sistema Nacional de Evaluación, Acreditación y Certificación de la Calidad de la Educación. Normas de competencia del profesional técnico en Contabilidad. Recuperado de: http//cir@sineace.gob.pe/www.sineace.gob.pe.

Vázquez, I., y Saitua, A. (2007). El desarrollo de competencias propuesta para la formación en el área contable del nuevo grado en relaciones laborales y recursos humanos de la UPV/EHU. Revista de Relaciones Laborales, 17(2), p. 139-162. Recuperado de: http//ojs.ehu.eus.

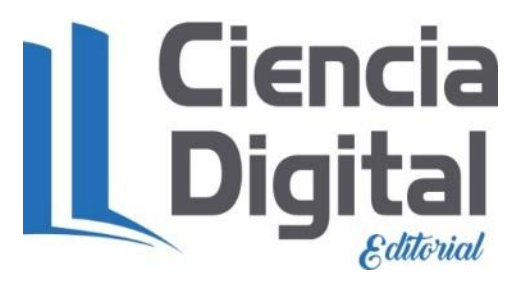




\section{PARA CITAR EL ARTÍCULO INDEXADO.}

Prado Chaviano, E., Montalvo Palacios, I., Celestrín Penabades, C., Arencibia Díaz, L., \& Velastegui López, L. E. (2021). Contribución del ejercicio integrador simulado para la formación inicial de la competencia profesional registrar hechos económicos. Visionario Digital, 5(2), 170-188. https://doi.org/10.33262/visionariodigital.v5i2.1710

\section{Ciencia \\ LDigital}

El artículo que se publica es de exclusiva responsabilidad de los autores y no necesariamente reflejan el pensamiento de la Revista Visionario Digital.

El artículo queda en propiedad de la revista y, por tanto, su publicación parcial y/o total en otro medio tiene que ser autorizado por el director de la Revista Visionario Digital.
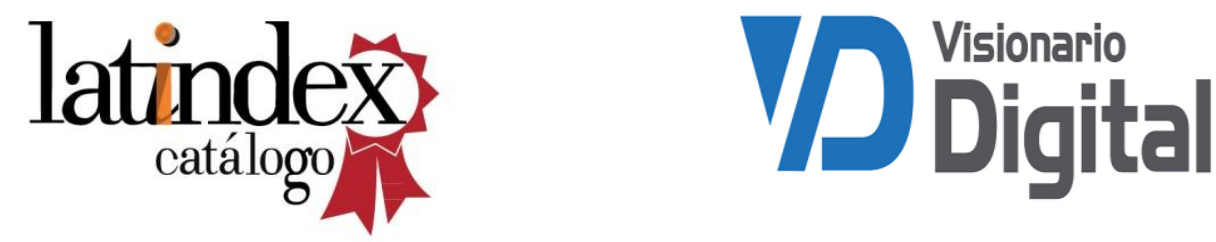